\title{
Best-fit criterion within the context of likelihood maximization estimation
}

\author{
S. Aranda ${ }^{\mathrm{a}, *}$, J.M. Linares ${ }^{\mathrm{b}}$, J.M. Sprauel ${ }^{\mathrm{b}}$
}

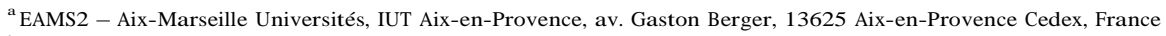

b ISM/GIBO UMR6233 - Aix-Marseille Universités, IUT Aix-en-Provence, av. Gaston Berger, 13625 Aix-en-Provence Cedex, France

\begin{abstract}
This article presents an approach toward surface best-fit based on Bayesian inference sta-tistics. The objective is to propose a best-fit criterion which integrates the distribution of points around the best-fit feature. This approach is used to guarantee a better estimate of the best-fit feature parameters. The best-fit criterion proposed in this article accounts for varied distributions that are not necessarily symmetric, such as those generated by turning and milling processes. It forms a generalization of least squares and enables the user to add information concerning the expected residual distribution shape. Therefore it provides the same results as the least squares method when the hypothesis of normal distribution is chosen. This article shows that using the proposed criterion will bring about a better estimate of the orientation of the best-fit feature and will lead to an evaluation of the form defect which is the closest to actual fact.
\end{abstract}

Keywords: Best-fit Criterion

Likelihood maximization Unsymmetrical, Density function

\section{Introduction}

This article focuses on surface best-fit within the framework of mechanical parts checking. The best-fit process aims to build mathematical models which represent real surfaces. It is based mainly on the determination of feature mathematical models, the use of best-fit criterion and the measurement of points set by coordinate measuring machines (CMM). The best-fit criterion is a key element in the process. Its role is to estimate the parameters of the mathematical models according to the coordinates of the sets of points. The evolution in manufacturing processes over the past few decades has brought about a great increase in the geometric quality of mechanical systems. Tolerance levels and, indirectly, conformance zones are decreasing as a consequence. The checking process has been forced to adapt to this evolution. Today, the use of a mathematical model representing a surface is no longer satisfactory. It is also necessary to have a knowledge of the deviation which exists between this model and the real surface. It permits qualifying and quantifying the uncer- tainty of the parameters of the obtained model. As a result, mastering this deviation has become one of the major stakes of modern metrology. Brought about through the evolution in the means of measurement and by integrating statistical concepts, a great number of approaches to surface best-fit incorporating the notion of uncertainty have been proposed over the last twenty years.

This article will attempt to add new elements as a response to the necessity of mastering the deviation between a surface and its best-fit mathematical model by using a statistical point of view of the best-fit process. The aim is to propose a best-fit criterion capable of accounting for the distribution of real surface points around an associated feature. This approach has proven to be interesting as it can simultaneously quantify the parameters of the associated feature as well as the deviation between this feature and the real surface.

This article has been divided into four parts. In the first part, the article will deal with different paths enabling control over deviation between the surface and its mathematical model. Existing approaches will be discussed and classified. The following section will sketch out the basis of the best-fit approach that we propose and will introduce a new best-fit criterion based on the use of likelihood 
maximization estimation (LME). The third and final sections will be devoted to the implementation of the proposed best-fit criterion and the analysis of the results obtained. As a conclusion, the article will end by reviewing the objectives reached and detailing future prospects.

\section{State of the art of best-fit approaches}

\subsection{Main directions in research}

Two points of view stand out among the numerous approaches that are proposed to solve the problem of surface best-fit. The first point of view considers the best-fit process as a purely geometrical operation. The goal is to minimize a function of the distances between the sampled surface and the best-fit feature. These distances are often called residuals. The proposed solutions generally consist in making complex the mathematical representation of the feature until weak and purely random residuals are obtained. This point of view is interesting insofar as it provides additional information about the topology of the best-fit feature (form, undulation, etc.) and insofar as it generates a Gaussian distribution of uncertainty. With the second point of view, the best-fit process is considered as a statistical approach similar to regression analysis. The objective is not to reduce the deviation between the bestfit feature and its mathematical representation but to use it as a source of information. In the end, a representation of the real surface and not the sampled surface can be determined. In order to achieve this, the deviation between the real and nominal geometries must be modeled in the form of a probability density function (PDF). Thus, a link between the knowledge of the measured coordinates and the points of the real surface can be established. The main interest in this point of view come from it ability to determine a representative feature of the real surface.

Whatever point of view is favored, every approach aimed at improving surface best-fit is confronted with the necessity of integrating more information about the deviation between the real and nominal features. Two strategies are used to achieve this goal. The first one consists in increasing the number of parameters describing this deviation, at the risk of generating additional costs in terms of calculations. According to the statistical or deterministic point of view; a line will be replaced by a polynomial equation of $\mathbf{n}$-degree or a Fourier series in order for all measurable geometric flaws to be describable; a PDF will be modeled by a general law such as a beta one. The second strategy consists in using trade knowledge to predict this deviation in a significant and sufficient way. As a function of the manufacturing parameters, perturbations are added to the geometrical best-fit feature or to the PDF. They come from a catalog of elementary deviations.

The illustration in Fig. 1 establishes a classification of the approaches and research work in the form of a reference system. The horizontal axis shows the adopted point of view. The vertical axis differentiates the strategies in deviation modeling. All in all, four distinct orientations, each corresponding to a quadrant of the referential system, are highlighted by this graphic representation. The

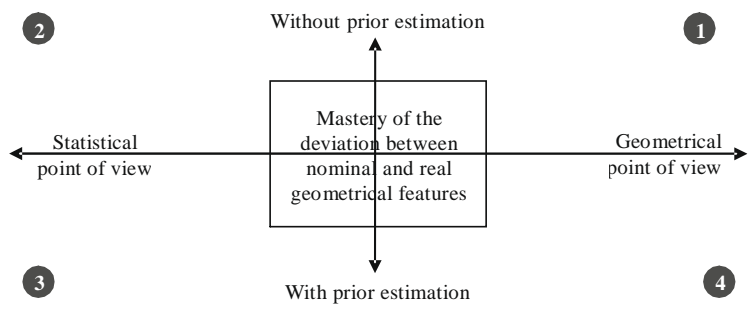

Fig. 1. Synthesis of the approaches used to control deviation with the real surface.

following paragraph will give a detailed explanation of these approaches and will present different research papers that fall within their scope.

\subsection{Classification of current approaches}

Quadrant 4 of the referential system (Fig. 1), shown in detail in Fig. 2, corresponds to an approach based on a geometrical point of view. Kurfess and Banks [1] propose a method resting upon the implementation of a catalog for cylindrical surface defects: tri-foil, diabolo, etc. The most likely geometrical deviations set is selected using a rule based on LME. It leads to the building of a mathematical model which is the closest possible to the real geometry.

The Henke and Summerhays approach [2,3] distinguishes itself from the previous model in the simultaneous integration of every geometric deviation into the surface mathematical model. This model is made up of the nominal geometry, here a cylinder, to which a perturbation $f(h, z)=a_{1} f_{1}(h, z)+a_{2} f_{2}(h, z)+\ldots+a_{n} f_{n}(h, z)$ is added. This modifies the value of the radius as a function of the angular position (h) and the position on the axis of the cylinder $(z)$. Functions $f_{i}$ characterize a series of elementary corrections whose effects are weighted by scalars $a_{i}$. The authors propose an implementation of this method based on the functions $f_{i}$ of the Fourier series, Eigen shapes and also polynomial equations. Killmayer and Babu [4] adopt a very similar approach to the one proposed by Kurfess and Bank. The originality of this approach comes from the implementation of a genetic algorithm which ensures the selection of the most pertinent geometric deviations.

Quadrant 1 of the referential system proposed in Fig. 3 focuses on a geometric point of view without any prior knowledge. This point of view consists in building a generic model of the surface which is able to describe any surface geometry. Tilo Pfeifera et al. [5] proposes an identification method of the geometry based on the topog-

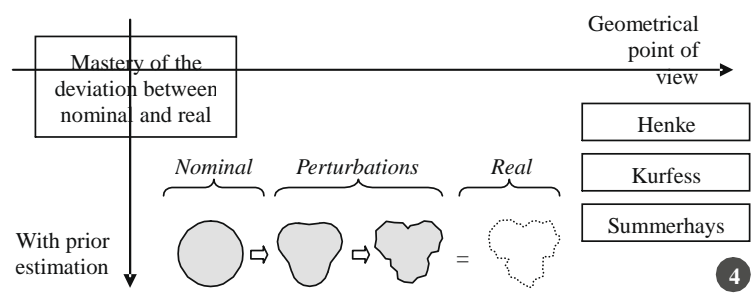

Fig. 2. Geometrical point of view with prior estimation. 


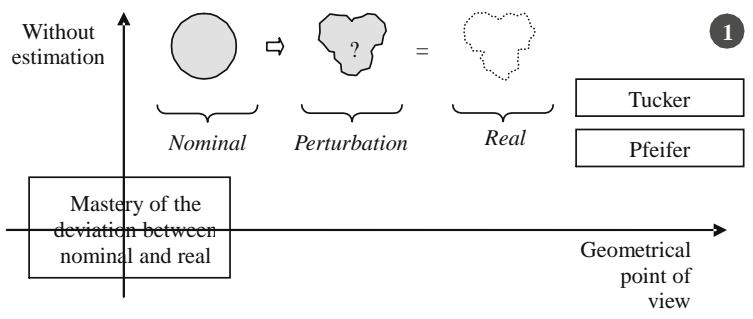

Fig. 3. Geometrical point of view without prior estimation.

raphy decomposition. The real geometry is divided into three generic shapes, each one associated with a scale of magnitude. This geometric representation obviously requires the establishment of a mathematical definition of the three deviations in shape, but also specific best-fit criteria. ${ }^{A}$ for Tuck $\mathrm{r}$ and Kurfess [6,7], they developed a method of best-fit parametric surfaces. This was carried out by stretching the analytical approach as far as possible. This type of advance has shown itself to be important since research based on a deterministic point of view without estimations is not currently being explored to any large extent because of the enormous calculation costs.

Quadrant 3 of the referential system proposed in Fig. 4 is founded on a statistical point of view with prior estimations of deviations. Choi and Kurfess [8] propose a study on the determination of uncertainty relative to shape deviation as a function of probed points. They propose treating form defect like a random variable sensitive to surface sampling. To evaluate this uncertainty, the authors underline the necessity of starting by modeling the distribution of the points of a machined surface around its nominal geometry. They advise against using a Gaussian law in this context. According to the authors, beta distribution appears to be a much better candidate in this specific case. It corresponds ideally to the description of plateau-type profiles and can, if necessary, give a rough estimate of the uniform or Gaussian distributions by making a judicious choice of parameters. Mestre $[9,10]$ was interested in the reconstruction of missing information when measuring with CMM. This kind of measurement only gives partial information on the surface geometry. To counteract this problem, Mestre underlines the necessity of resorting to inference statistics and creating a catalog of geometric deviations.

Quadrant 2 of the referential system proposed in Fig. 5 carries on with the statistical approach but does without prior knowledge. Continuing on the research discussed

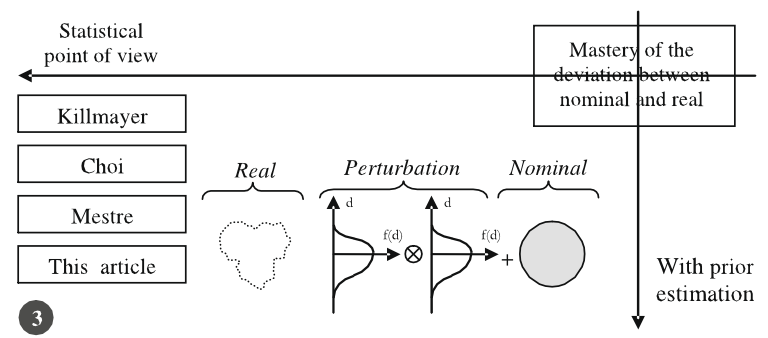

Fig. 4. Statistical point of view with prior estimation.

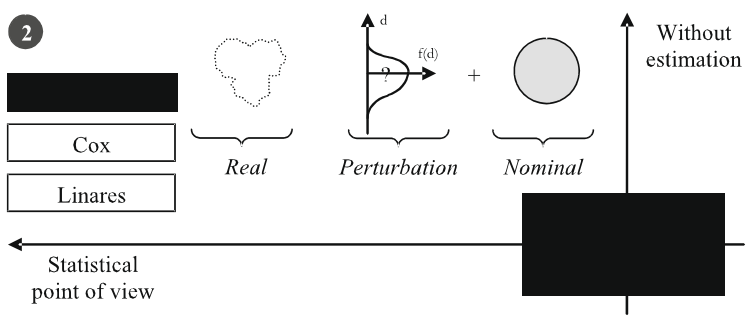

Fig. 5. Statistical point of view without prior estimation.

above, Choi and Kurfess 11] presented a method of determining uncertainty on the exterior tangent feature based on the BOOTSTRAP technique. Linares et al. [12,13] proposed a method of determining uncertainty on the bestfit feature using the least square criterion (LSC). As for Cox $[14,15]$, he was interested in using the LSC in cases when residual distribution is not necessarily Gaussian. He proposes first using the $\mathrm{v}^{2}$ test in order to estimate least square conformity. In case this appears to be unsatisfactory, he suggests solving the problem by modifying data, the model or even by distorting input data.

\section{Best-fit method within the context of LME}

A great number of approaches underline the importance of the distribution of points making up the real surface around the best-fit feature: either to determine uncertainty or in the selection of criterion. For example, Kurfess proposes modifying the input data to guarantee an adapted residual distribution. It is this adaptation of distribution which guarantees that the calculated feature truly describes the real surface and not just the point sample.

Another solution may be to propose a best-fit criterion which imposes no particular hypothesis on point distribution. The criterion should not try to correct PDF but uses it as it comes as a source of information. It is this last path that has been chosen.

\subsection{Best-fit principal within the context of LME}

The approach proposed in the next pages belongs to quadrant 3 of the referential system (Fig. 4). It rests upon a statistical point of view of surface best-fit.

The surface is considered to be an unknown population made up of infinite points. The only source of available information about it is a sampling of measured points. The purpose of best-fit surfaces is to estimate the real surface's geometric characteristics (position and orientation) from this sampling. It is important to note that the objective is to determine the features of the real surface and not those of the representative surface of the point samples. Determining extreme-fit in the usual way by metrology software calculations does not meet this objective. It is based on the hypothesis that extreme points in the sample measurements are representative of extreme points on the real surface. This hypothesis is not realistic if the small number of evaluated points is taken into consideration. Only the extreme-fit to the sample can, in these conditions, 


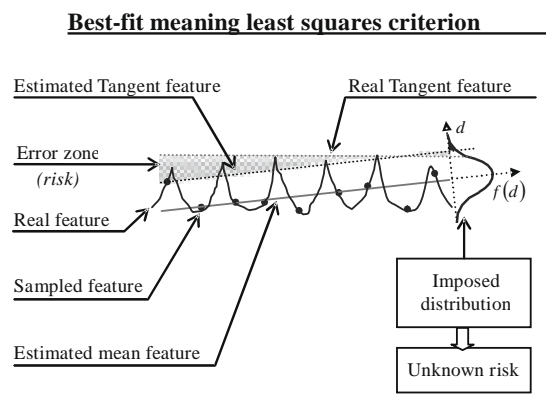

Tangent feature only determined with one point. Orientation determined with the sampled

Risk is unknown and not reliable.
Best-fit meaning likelihood maximization

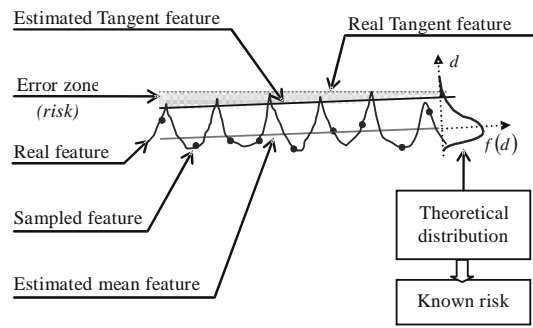

Tangent feature and orientation determined with the sampled points and information onto the real surface topology

Risk is known and reliable.

Fig. 6. Principle and interest of the likelihood maximization approach.

be determined. To apprehend extreme-fit, the infinite points making up the real surface must be worked on. If these are unknown, the only available data is an estimation $\bar{f} \partial \mathrm{xp}$ of their distribution around the mean surface. The extreme point cannot be apprehended with this data, but using the data offers the possibility of determining, with a given risk, a limit beyond which points should not exist (Fig. 6).

The distribution $\mathrm{f}(\mathrm{x})$ of points around the mean surface is mostly imposed by the manufacturing process used to generate it. It represents a signature, translating the topology imposed on the surface in statistical form, through the movement of generation and the cutting-edge geometry of the tool. It can thus be estimated from the knowledge of the manufacturing conditions of the surface.

This estimation can be obtained in several ways: creating an experimental catalog of PDF, training a neural network to estimate the parameters of a generic PDF or running a machining simulation. This aspect will not be dealt with in this article. It will be taken for granted that it is always possible to obtain an estimation of the distribution of points making up the real surface around the nominal geometry. This estimated distribution will be marked $\overline{\mathrm{f}} \mathrm{\partial x}$ in order to not be confused with the real distribution $f(x)$ which cannot be known. As many researchers have underlined, the shape of $\bar{f} \partial \mathrm{xP}^{\mathrm{P}}$ is principally a function of the manufacturing process used. A surface of very good quality will present a symmetrical distribution with a normal tendency, whereas a turned or milled surface will present an asymmetrical and possibly multimodal distribution.

The importance of accounting for the signature of the manufacturing process is not only limited to determining extreme-fit. As shown in Fig. 6, best-fitting with a criterion who imposes a distribution which is non-representative of distribution of the surface can also lead to a poor estimation of the parameters of the best-fit feature. A good estimation can be obtained by considering the surface bestfit as a regression analysis. The goal is, in this case, to determine the parameters of the best-fit geometry which maximizes likelihood between the residual best-fit distribution and the theoretic signature of the surface. Through this approach, inference statistics properties can be used. The best-fit method is comprised of three steps (Fig. 7). First of all, an initial estimate of the distribution shape of points is determined as a function of manufacturing parameters. This is called prior distribution because it consists in a hypothesis of residual distribution. Next, a best-fit criterion is applied to determine parameters which maximize likelihood as a function of the predicted distribution. The parameters determined by the criterion are the position of the best-fit feature, its orientation, its possible intrinsic parameters, as well as the mean and standard deviation of the prior distribution. This leads to the final

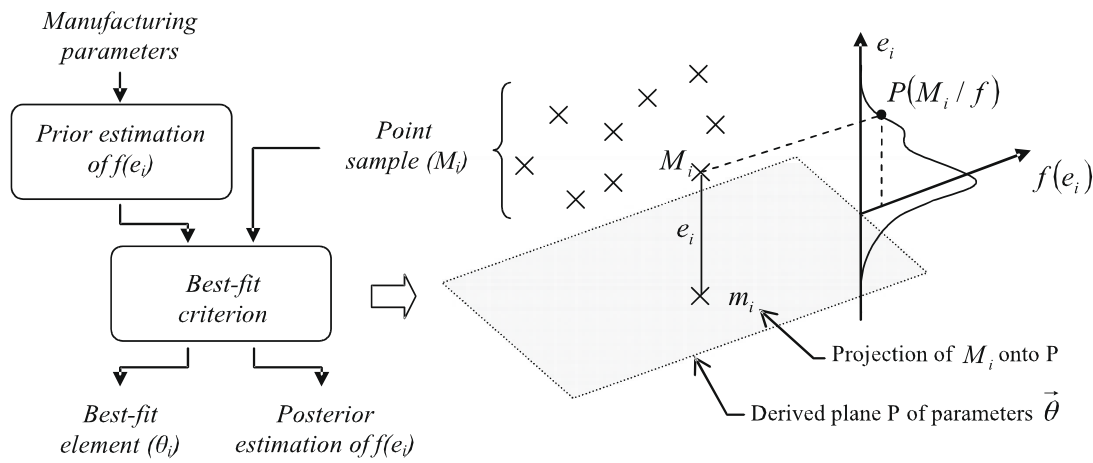

Fig. 7. Principle of a best-fit criterion based on LME 
step, where a new estimate of distribution is made as a function of the parameters calculated in the previous step It is called posterior distribution because it takes into account the knowledge included in the points sample. These last two steps may be repeated if the prior and posterior PDFs are too far apart. The following paragraph introduces a best-fit criterion within the context of the LME which represents the key element in this approach. This criterion will be logically called as maximum likelihood criterion (MLC).

\subsection{MLC: best-fit criterion based on LME}

The first step, in the development of a MLC, consists in determining the cost function CðП optimum parameters of the best-fit model should be obtained. Using the parameterization proposed in Fig. 7 and considering points $M_{i}$ as independent, the cost function of the estimate is written:

Cðగp 1/4 ${ }_{i 1 / 41}^{Y} \mathrm{P \partial M}_{\mathrm{i}}=\mathrm{fP}$

It can be rewritten as a function of the cumulative distribution function $\mathrm{f}\left(\mathrm{e}_{\mathrm{i}}\right)(\mathrm{CDF})$ :

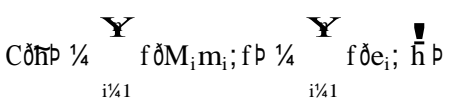

This new formulation of the cost function is often called the likelihood function. The optimum parameters sought out are those which maximize this function, seeking out the best-fit feature amounts to solving the equation:

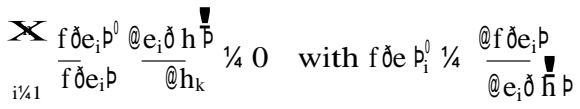

Because this equation cannot always be solved directly, it is interesting to linearize it by replacing $\mathrm{f}\left(\mathrm{e}_{\mathrm{i}}\right)$ with its Taylor limited development to the second order:

$f \partial e_{i} p 1 / 4 f \partial e_{i 0} p p f \partial e_{i 0} p^{0} \partial e_{i} \ddot{y} e_{i 0} p 1 / 4 f^{\chi} e_{i 0} p p f \partial e_{i 0} p^{0} d e_{i}$ with $\mathrm{de}_{\mathrm{i}} 1 / 4 \partial \mathrm{e}_{\mathrm{i}} \ddot{\mathrm{y}} \mathrm{e}_{\mathrm{i} 0} \mathrm{p}$

Thus rewritten, the equation to be solved becomes:

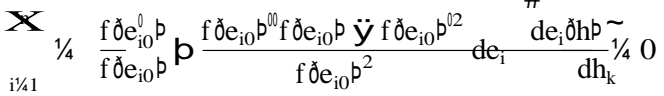

This equation is particularly interesting, because it can be reduced to weighted least squares:

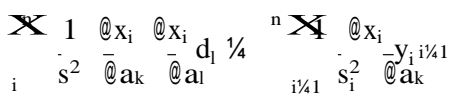

By setting out:

$$
\begin{aligned}
& \mathrm{x}_{\mathrm{i}} 1 / 4 \mathrm{e}_{\mathrm{i}} ; \quad \mathrm{a}_{\mathrm{k}} 1 / 4 \mathrm{~h}_{\mathrm{k}} ; \quad \mathrm{s}_{\mathrm{i}}^{2} 1 / 4 \frac{\mathrm{f} \partial \mathrm{e}_{\mathrm{i} 0} \mathrm{p}^{2}}{\mathrm{f}^{2} \mathrm{e}_{\mathrm{i} 0} \mathrm{p}^{0} \mathrm{f} \mathrm{fe}_{\mathrm{i} 0} \mathrm{p} \ddot{\mathrm{y}} \mathrm{f} \partial \mathrm{e}_{\mathrm{i} 0} \mathrm{p}^{02} ;} \\
& y_{i} 1 / 4 \frac{f^{\partial} e_{i 0} p f \partial e_{i 0} p^{0}}{f \partial e_{i 0} p^{00} f^{\partial} e_{i 0} p \ddot{y} f \partial e_{i 0} p^{02}}
\end{aligned}
$$

This analogy is very practical since it enables the use of the same procedures leading to a solution as those developed in the framework of the LSC.
It is also possible to show the equivalence between the proposed criterion and the LSC when the imposed distribution shape $f\left(e_{i}\right)$ is normal. Using the hypothesis of a Gaussian residual distribution, it is possible to write the imposed distribution as:

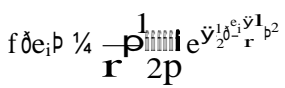

where $\mathbf{1}$ is the mean and $\mathbf{r}$ is the standard deviation

By using this expression into the definition of the variables equivalent to LS, which were obtained in the above paragraph, it is easy to show that the following is obtained:

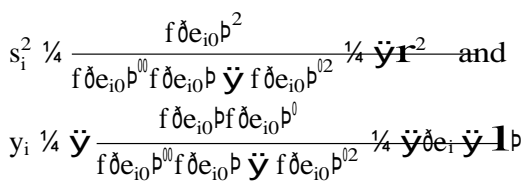

These equalities demonstrate that the proposed criterion is equivalent to the LSC using the hypothesis of a normal residual distribution. This equivalence makes possible using the same solving method than the many ones developed for LSC.

\subsection{Method of estimating the imposed distribution $\bar{f} \partial x p$}

As the followed objective is taking into account any distribution shape, a generic formulation of the imposed PDF $\overline{\mathrm{f}} \mathrm{\partial x}$ has to be developed. Three constraints should be respected in this formulation:

Considering the prediction model of the distribution used in our best-fit method, this function $\bar{f} \partial x p$ should be derived from a set of values forming a sampling of distances between the nominal surface and the theoretically manufactured surface.

Because of the expression of the proposed criterion, the model chosen must also give a good estimation of the first and second distribution derivatives of $\overline{\mathrm{f}} \mathrm{\partial x}_{\mathrm{x}}$.

In order to ensure the convergence of the criterion, the model of function $\overline{\mathrm{f}} \partial \mathrm{xP}$ must lead to a coherent estimation of the probability of observing a distance $x$ as much within the definition interval of $x_{i}$ as outside it.

A first approach would consist in approximating the PDF by a histogram. In other words, it consists in estimating the density $\overline{\mathrm{f}} \mathrm{\partial x}$ of the random variable $x_{i}$ in relation to the ratio $n_{i} / N$, where $n_{i}$ represents the occurrences of $x_{i}$ belonging to the $i^{\text {th }}$ class associated to a given value $x$ and $\mathrm{N}$ represents the total number of realizations. As the density is thus the same whatever the position of $\mathrm{x}$ between the extremities of this class, this approach does not guarantee the convergence of the best-fit procedure. It does not provide a continuous and regular estimate of density. For this reason, it has been abandoned and replaced by a more effective estimation method.

An improvement consists in using a mobile window around value $x$ and a density estimation function $K(u)$, called core. The estimation of the probability of observing value $\mathrm{x}$ knowing $\mathrm{n}$ of its $\mathrm{x}_{\mathrm{i}}$ realizations is given by: 


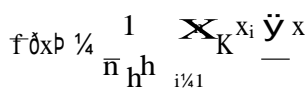

Variable $\mathrm{h}$ is called the window opening factor. It determines the level of fitting operated by the core. The greater its value, the more the probability function is best-fitted and the less informative it becomes. The optimal value of $\mathrm{h}$, which leads to an evaluation that is both regular and sufficiently detailed, is generally close to $n^{\ddot{y}^{1 / 5}}$.

The preceding equation determines the probability of observing a value as a function of several realizations of the random variable. In this case, each realization has as many chances of occurring as another. It is also possible to express the probability of observing a value as a function of $n$ probabilities of observing particular $x_{i}$ values. For this to happen, the equation must contain the probability $\mathrm{p}\left(\mathrm{x}=\mathrm{x}_{\mathrm{i}}\right)$ of observing a value $\mathrm{x}_{\mathrm{i}}$, noting that the number of occurrences of value $x$ must, theoretically, be observed for $\mathrm{N}$ realizations is $\mathrm{n}_{\mathrm{i}}=\mathrm{N} \mathrm{p}\left(\mathrm{x}=\mathrm{x}_{\mathrm{i}}\right)$. The total number of observations is equal, in this case, to $n 1 / 4 N_{i^{1} / 41}$ pox $1 / 4 x_{i} p$ and the expression of the probability function becomes:

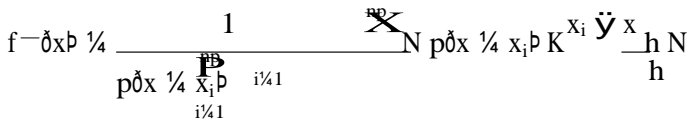

By eliminating the total number of $\mathrm{N}$ realizations, we obtain an alternative formulation of $\bar{f} \partial x p$ which is a direct function of the probabilities of observing $n p$ values $x_{i}$ :

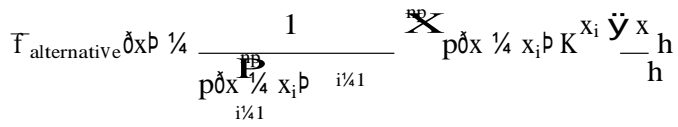

One or the other expressions, $\overline{\mathrm{f}}$ or $\overline{\mathrm{f}}_{\text {alternative }}$, can be used depending on the scenario. The first expression will be used to construct an estimate as a function of a set of values obtained through a simulation of machining. The second one will be used to construct an estimate from a known law in order to apply a standard criterion. To guarantee the derivability and continuity of the estimate, the choice was made to use a Gaussian core:

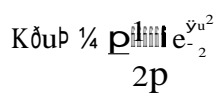

Using this type of core entails a slightly Gaussian shape of the estimated probability function. This shape, generated by the core, is not penalizing, however. The PDF which describes the distribution of measured points is the result of the convolution between the manufacturing signature and that of the CMM. Since the latter is generally Gaussian, the effect of this fitting is in line with a coherent hypothesis on the prior PDF. Another advantage in the formulation of the core that has been retained is that it guarantees one of the conditions for the existence of $\bar{f} \partial x$ :

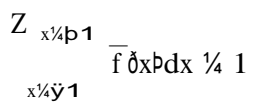

In the end, Gaussian core-fitting is an interesting way for estimating the function $\mathrm{f}(\mathrm{x})$ :

it builds a continuous and regular estimate of PDF directly from a points sampling,

it can be effective with a reasonable number of points, it ensures the convergence of the best-fit criterion by guarantying the existence of a weak and non-zero estimate of the probability of observing a point outside of the interval in which the surface points belong.

Two applications have been carried out to evaluate the efficiency of core-fitting. The first application consists in the construction of an estimate by core-fitting of a known law thanks to its formula. This enables us to implement a classic criterion (LS, uniform). The second application consists in estimating a known law with a set of deviations between the nominal surface and the theoretically generated one. The later is the most interesting way. It permits to implement a criterion with a distribution estimate obtained through a machining simulation.

Figs. 8 and 9 correspond to the first application. They present PDFs constructed from a discretized distribution of $\mathrm{N}=200$ ordered pairs $\left(\mathrm{x}_{\mathrm{i}} ; \mathrm{p}\left(\mathrm{x}=\mathrm{x}_{\mathrm{i}}\right)\right)$. The $\mathrm{x}_{\mathrm{i}}$ ordinates have been placed at regular intervals between $x_{\min }=\ddot{y} 5$ and $\mathrm{x}_{\max }=5$ and the corresponding probabilities $\mathrm{p}\left(\mathrm{x}=\mathrm{x}_{\mathrm{i}}\right)$ have

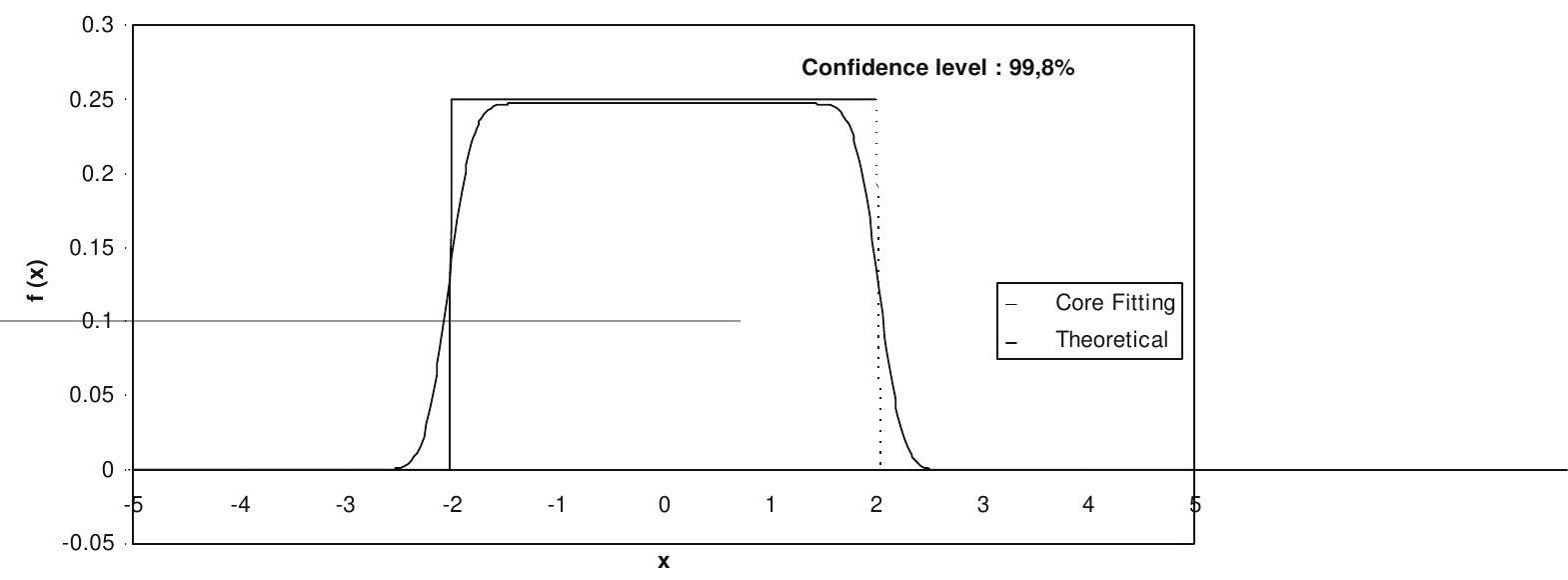

Fig. 8. Core-fitting using a uniform law $U[0,1 / 3]$ of 200 points 


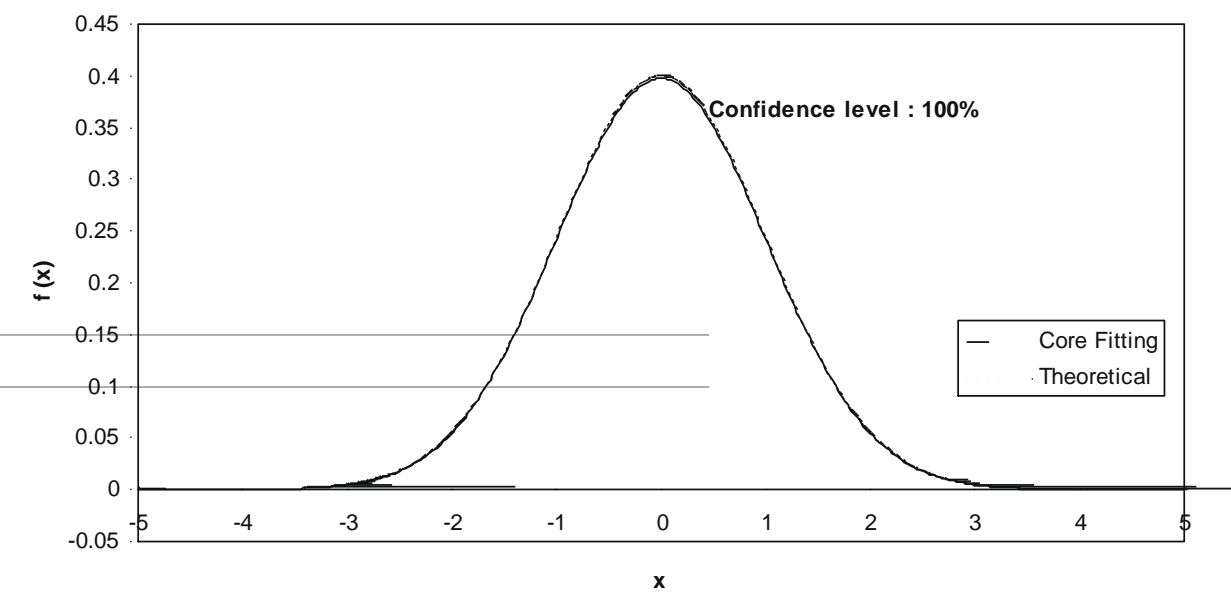

Fig. 9. Core-fitting using a normal law N[0, 1] of 200 points.

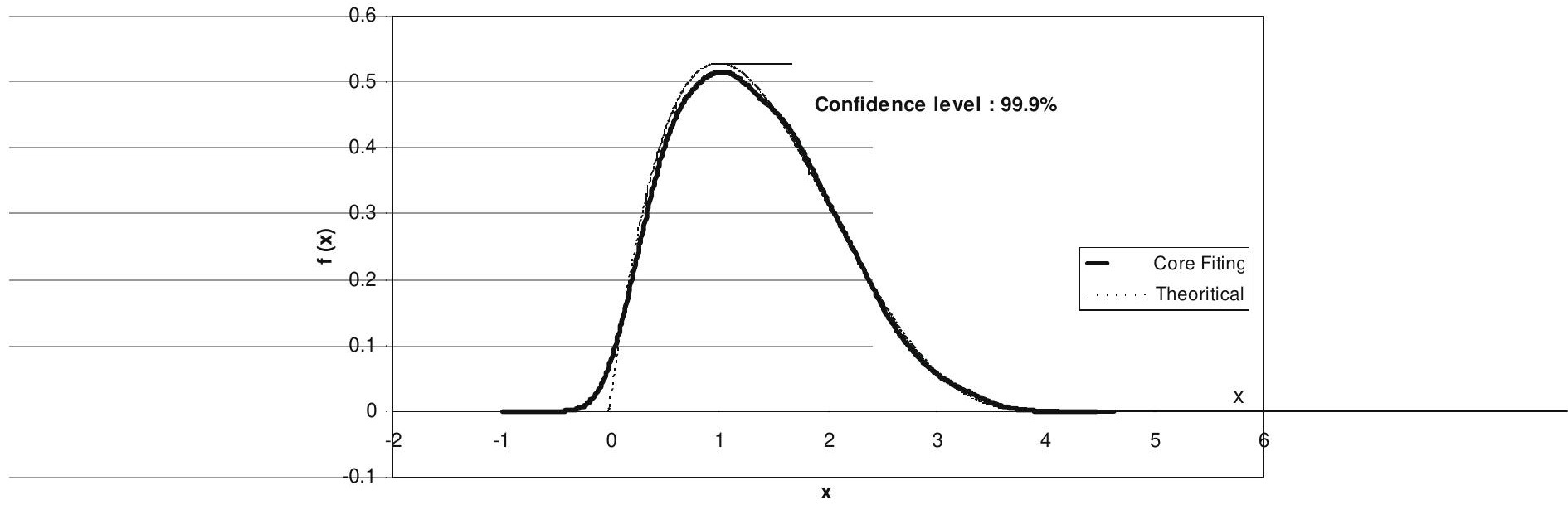

Fig. 10. Core-fitting using a beta law $\mathrm{B}[2,4]$ of 4000 points defined in the interval $[0,4]$.

been evaluated from the theoretical law. Reconstruction was obtained by using the alternative distribution estimate $\overline{\mathrm{f}}_{\text {alternative }}$ (Eq. (12)). In order to evaluate fitting efficiency, a Chi-square test was carried out for each reconstructed PDF. The test result is displayed in the Figures. It indicates the percentage of chances that the initial or reconstructed distributions match. Core-fitting can thus be seen as effective in these cases.

Fig. 10 presents the PDF reconstructed from a sampling of simulated values thanks to the Monte Carlo method (MCM) according to a known law. A sampling of 4000 was generated. In this Figure, the thin dotted line shows the shape of the law retained for this paper (a beta law $\mathrm{B}[2,4])$. Superposed on this line is a thick solid line showing the estimate reconstructed from the proposed fitting method accounting for the 4000 points available and in using the formulation of $\overline{\mathrm{f}} \mathrm{\partial} \mathrm{P}$ described by Eq. (10).

In view of the body of results, fitting with Gaussian cores seems to lead to a good PDF estimate. Results, not commented here, show that a minimum of 50 points taken by MCM is necessary in order to reach a suitable estimate and that an optimum between estimation quality and calculation time appears to be around 200 points.

\section{Validation and efficiency with the LSC}

4.1. Validation of the equivalence with the LSC

The equivalence between the MLC and the LSC was demonstrated in Section 3.2. This equivalence, which exists as long as prior distribution is normal, has been used to validate the best-fit method proposed. A great number of point samplings have been successively associated with the both criteria and compared two by two. The mean values of the parameters determined in the two scenari only differ by $10^{\ddot{y} 9} 1 \mathrm{~m}$ for a distribution defined by 200 points.

\subsection{Estimation efficiency for large samplings}

The method proposed in this article was devised so as to be able to account for non-Gaussian and asymmetric 
distributions. It is important to check its ability to identify surface parameters as a function of prior distribution. when the later is fully known. To evaluate this aspect, two files of 1681 points were generated by MCM. Each file simulates the measurement of a plane of normal $\bar{Z}$, of an area of $40140 \mathrm{~mm}$ and presenting a form-error of $0.1 \mathrm{~mm}$. Each file presents a distinctive distribution of deviations following $\mathrm{z}$. The first file presents a uniform distribution U[ÿ0.05, 0.05] and the second one shows a dissymmetric distribution following a beta distribution $\mathrm{B}[2$, 5].

File 1 has been successively processed with the LSC and the MLC based on the appropriate hypothesis of uniform distribution U[ $[\ddot{y} 0.05,0.05]$. Table 1 presents the:

values of the solid angle between the normal to the bestfit surface and the axis $\bar{z}$,

form defect (some flaws in shape) present in the residual,

standard deviation in calculated residuals for each bestfit scenari.

It also indicates the theoretical values (truly present in the files) and the error resulting from each criterion. The cases shown in bold character indicate the most accurate results.

The solid angle is well evaluated by the two criteria. The error of the LSC is only about $61 \mathrm{~m} / \mathrm{m}$. The form-error is evaluated adequately by the MLC leading to an error of $0.00041 \mathrm{~m}$ compared to $3 \mathrm{1m}$ for the LSC. Standard deviation is poorly evaluated in both cases. The criteria have an error of $121 \mathrm{~m}$.

File 2 has been successively processed with the LSC and the MLC based on the appropriate hypothesis of a beta distribution $\mathrm{B}[2,5]$. Table 2 below presents the results obtained for this file:

The solid angle is well evaluated by the two criteria The error induced by MLC is once again the lower than $0.00021 \mathrm{~m} / \mathrm{m}$. The form-error is well evaluated by the MLC. The error on the estimated form-error is $0.061 \mathrm{~m}$ for the MLC compared to $0.21 \mathrm{~m}$ for the LSC. Standard

Table 1

Comparison of the results of best-fit for a uniform distribution.

\begin{tabular}{|c|c|c|c|}
\hline & Solid angle/z & Form-defect & $\begin{array}{l}\text { Standard- } \\
\text { deviation }\end{array}$ \\
\hline Theoretical & 0 & 0.1 & 0.016552014 \\
\hline LSC value & $9.34005 \mathrm{E} \ddot{y} 05$ & 0.102624175 & 0.028872132 \\
\hline MLC value & $6.35323 \mathrm{E} \ddot{y} 06$ & 0.100003992 & 0.02889064 \\
\hline LSC error & 9.34005E ̈̈ 05 & 0.002624175 & 0.012320117 \\
\hline MLC error & $6.35323 E \ddot{y} 06$ & $3.99163 \mathrm{E} \ddot{y} 06$ & 0.012338626 \\
\hline
\end{tabular}

Table 2

Comparison of the results of best-fit for a beta distribution.

\begin{tabular}{llll}
\hline & Solid angle/z & Form-defect & Standard-deviation \\
\cline { 2 - 3 } Theoretical & 0 & 0.1 & 0.016552014 \\
LSC value & $4.19013 \mathrm{E} \ddot{y} 05$ & 0.099795933 & 0.016544583 \\
MLC value & $6.77056 \mathrm{E} \ddot{y} 06$ & 0.099942699 & 0.01655385 \\
LSC error & $4.19013 \mathrm{E} \ddot{\mathbf{y}} 05$ & 0.000204067 & $7.43116 \mathrm{E} \ddot{y} 06$ \\
MLC error & $6.77056 \mathrm{E} \ddot{\mathbf{y}} 06$ & $5.7301 \mathrm{E} \ddot{\mathbf{y}} 05$ & $1.83591 \mathrm{E} \ddot{\mathbf{y}} 06$ \\
\hline
\end{tabular}

deviation is better evaluated than in the case of File 1. All the criteria have satisfactory results and the best estimate is obtained with the MLC which is off only by $0.0021 \mathrm{~m}$.

In view of these results, it is possible to conclude that the proposed criterion proves to be efficient in determining the geometric characteristics of best-fit features such as position and orientation. It is also possible to assert that it serves as a good estimator of form-error. However, the MLC does not seem to be the best estimator of standard deviation in residuals. It gives results that are similar to those of LS which only applies to the information contained in the sampling and not on the information from the real population. Additional research should be carried in this direction.

\subsection{Estimate efficiency for limited samples}

The previous paragraph showed how the proposed criterion could use the knowledge of theoretic residual distribution to draw out better additional information about the set of points. This information gives a better estimate of form-error when the imposed distribution and the distribution present in the sample of measured points match. This paragraph is concerned with the situation where the number of points representing the measured surface is limited and leads to a poor estimate of real distribution. To do this, sub-samples of 50, 200 or 300 points are sampled without replacement from the previous Files 1 and 2. They are best-fit with the method proposed and the LSC in order to estimate the form-error present on the surface. This form-error has been chosen as the subject of study because it is adequately estimated by the proposed criterion.

\subsubsection{The case of a set of points presenting uniform} distribution

The graph in Fig. 11 shows the form-error evaluated after best-fitting a series of 14 sub-samples of 300 points taken from File 1. Each sample has been best-fit with MLC (the solid line) and LSC (the thin line). The theoretical value of the form defect, shown by the thick line, is the one from File 1, i.e. $0.1 \mathrm{~mm}$.

It can be observed in this graph that MLC provides more stable and, globally-speaking, more accurate results. The mean error is about $0.751 \mathrm{~m}$ with a standard deviation of $0.67 \mathrm{Im}$ compared to five times that for the mean error committed by the estimate based on LSC. As for maximum error, it is not higher than $2.571 \mathrm{~m}$.

4.3.2. The case of a set of points presenting dissymmetric distribution $\mathrm{B}(2,5)$

The following graphs (Fig. 12) present the evaluation of the form defect obtained after best-fitting three series of 14 sub-samples taken from File 2. The three series are made up of samples consisting of 50, 200, 300 points respectively. The graphs are devised as in the former graphs and their analysis leads to the same conclusion: MLC proves to be a better estimator of form defects. An additional remark can be made. If the MLC is efficient in determining form defects, it is even better if the sample 


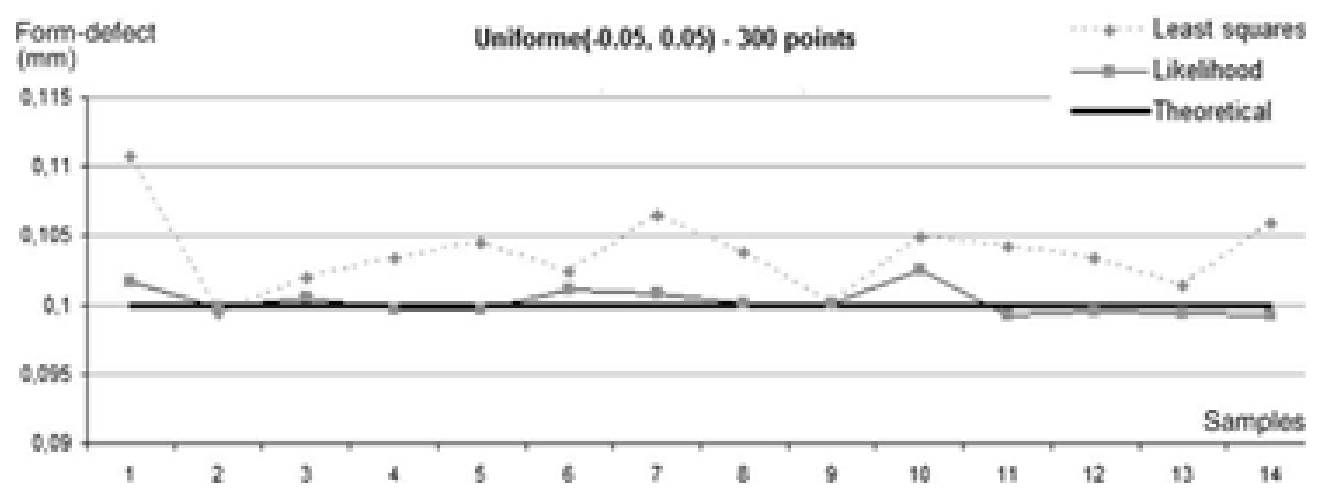

Fig. 11. Comparison between LSC and MLC results for a uniform distribution.

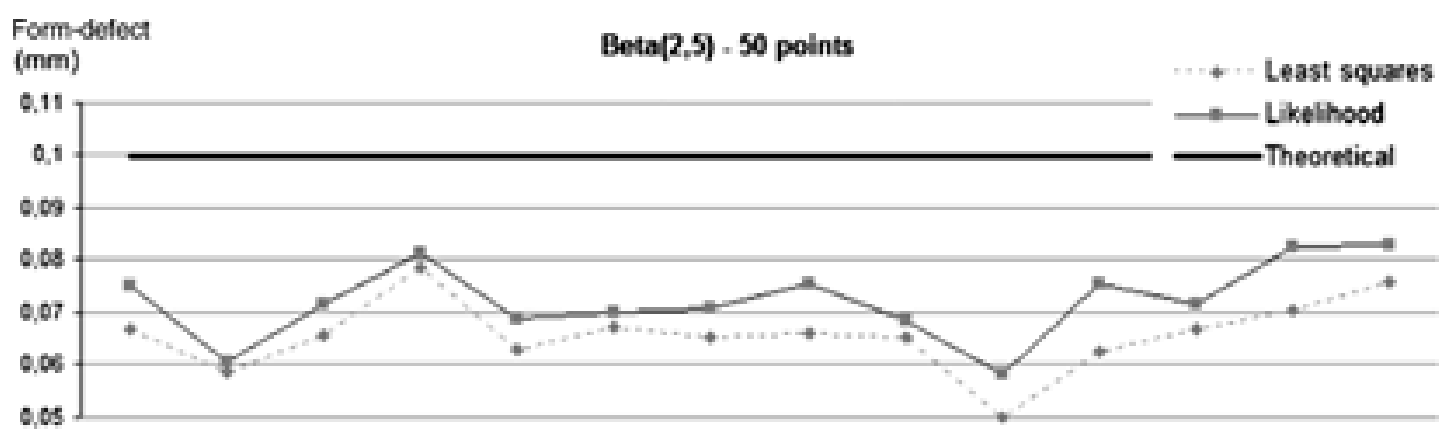

Form-defect
$(\mathrm{mm})$$\quad$ Beta[2,5) - 200 points

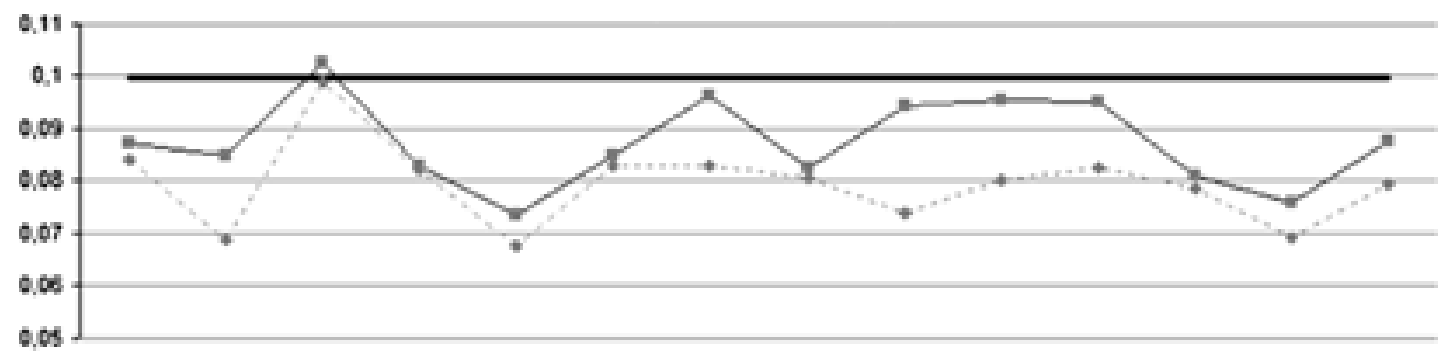

Form-defect

(mm)

Beta(2,5) - 300 points

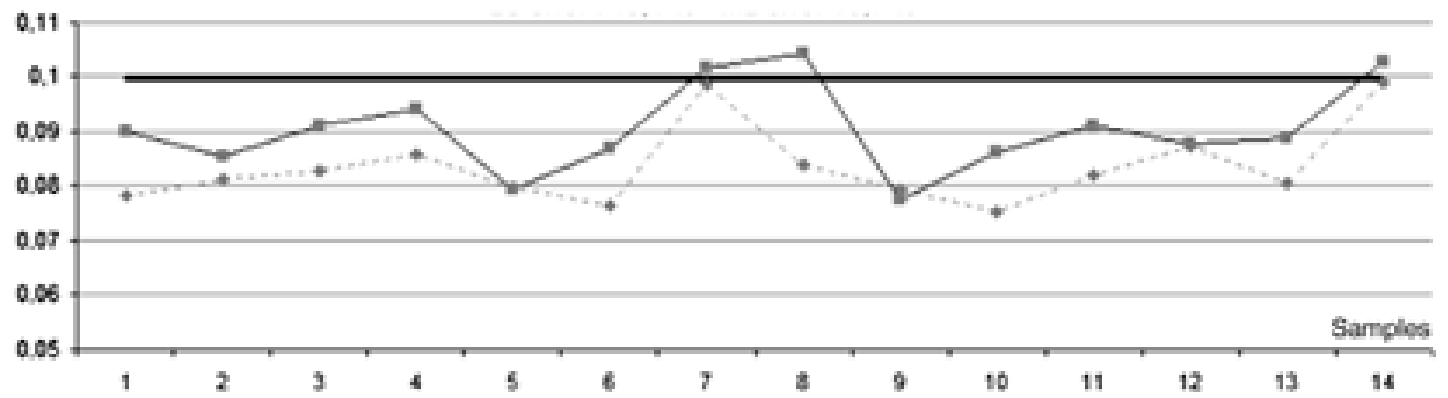

Fig. 12. Comparison of LSC and MLC results for a beta distribution.

is made up of at least a few hundreds of points. Thus, for very small-sized samples, the MLC is not significantly more efficient than the LSC. This comes from the fact that the sample does not contain enough information to make good use of distribution knowledge.
4.4. Efficiency of the criterion to maximize likelihood between residual and prior PDFs

A final idea for evaluating the relevance of the MLC lies in the analysis of best-fit residuals. The proposed criterion 
Table 3

Comparison of the likelihood of obtained residuals using LSC and MLC.

\begin{tabular}{|c|c|c|c|}
\hline Distribution & Criterion & Risk $\left(\mathrm{Ki}^{2}\right)$ & Recommendation \\
\hline \multirow[t]{3}{*}{ Normal } & LSC & $0.142 \mathrm{E}-$ & \multirow{3}{*}{$\begin{array}{l}\text { LS faster } \\
\text { estimator }\end{array}$} \\
\hline & MLC with & 16(same & \\
\hline & normal PDF & results) & \\
\hline \multirow[t]{3}{*}{ Uniform } & LSC & 0.870 & \multirow[t]{3}{*}{ ML best estimator } \\
\hline & MLC with & 0.109 & \\
\hline & uniform PDF & & \\
\hline \multirow[t]{3}{*}{ Beta } & LSC & 0.241 & \multirow[t]{3}{*}{ ML best estimator } \\
\hline & MLC with & 0.067 & \\
\hline & predicted PDF & & \\
\hline \multirow{2}{*}{$\begin{array}{l}\text { Slightly } \\
\text { bimodal }\end{array}$} & LSC & 0.830 & \multirow[t]{2}{*}{ ML best estimator } \\
\hline & $\begin{array}{l}\text { MLC with } \\
\text { predicted PDF }\end{array}$ & 0.058 & \\
\hline
\end{tabular}

aims to determine the best-fit feature so that the latter maximizes likelihood between the best-fit residual distribution and the theoretical signature of the surface. In other words, using this criterion must lead to the shape of residual distribution after best-fitting being roughly identical to the shape of the theoretical signature. If this will happen, it provides, obviously, that the hypothesis on the residual PDF is coherent. Nevertheless, the residual PDF here is necessarily founded, in light of the artificial generation of the point sample. To check the likelihood between the expected residual distribution and that obtained after bestfitting with the proposed criterion, four samples were generated from a virtually measured surface. Each plane has a known theoretical distribution in $\mathrm{z}$ direction: Gaussian for the first one, uniform for the second one, dissymmetrical unimodal for the third one and dissymmetric multimodal for the last one. Each sample was successively best-fit with the LSC and the MLC. The best-fit residual distributions were then compared with the known theoretical distributions. The comparison was made with a Ki-2 test applied to the central-reduced distributions, just accounting for the shape of the distributions. The risk linked to the hypothesis of non-fortuitous equivalence between the two distributions can be estimated. The lower is the risk the better is the agreement.

The table below sums up the results obtained. It shows that in any case, except for a Gaussian signature, the MLC leads to a more accurate estimate of the shape of residual distribution. In the case of a normal distribution, both criteria are equivalent and lead, quite naturally, to the same results. In this case, using the LSC is advised on the grounds of rapidity (Table 3).

\section{Conclusion}

This article introduced a new criterion for best-fitting geometrical features, called Maximum Likelihood criterion (MLC). This criterion is supported by the principles of LME and aims at better controlling deviation between the real surface and the best-fit geometry. Additional information about the shape of the theoretical distribution of points around the best-fit feature is introduced into the best-fit process in order to achieve the goal. This information is important as it enables better estimates of the parameters of the best-fit geometry.
The proposed criterion proves to be reliable when implemented. It demonstrated its ability to find the real signature of the surface, with relevance, and to be a good estimator of the orientation of the best-fit feature. It also proved to be pertinent in estimating form defects for the samples made up of a few hundreds of points.

Several important conclusions must be drawn from this research. First of all, using the LSC in situations where the residual distribution is not, a priori, Gaussian, leads to a non-relevant estimate of the residual distribution. A criterion based on LME appears to be a better solution only if there is a prior estimation of the shape of the real residual distribution.

Furthermore, it is important to note that no criterion has proven to be capable of providing relevant results on the estimation of surface form defect for samples of fewer than 100 points. Implementing the proposed criterion is only efficient with samples of a minimum of 200300 measured points. Under this threshold, there does not seem to be enough exploitable information in the sample.

Finally, it is interesting to note that the proposed approach marks a generalization of certain standard best-fit criteria. It is, for example, strictly equivalent to the LS when prior distribution is felt to be Gaussian. Moreover, it makes working with non-symmetric and slightly multimodal distributions possible.

New possibilities are opening for the determination and utilization of uncertainties linked to parameters estimated by the MLC.

\section{References}

[1] T.R. Kurfess, David L. Banks, Statistical verification of conformance to geometric tolerance, Computer-Aided Design 27 (5) (1995) 353 -

[2] R.P. Henke, K.D. Summerhays, J.M. Baldwin, R.M. Cassou, C.W. Broxn Method for part evaluation of systematic geometric deviations in machined part and their relationships to process variables, Precision Engineering 23 (1999) 273-292.

[3] K.D. Summerhays, R.P. Henke, J.M. Baldwin, R.M. Cassou, C.W. Broxn Optimizing discrete point sample patterns and measurement data analysis on internal cylindrical surfaces with systematic form deviation, Precision Engineering 26 (2002) 105-121.

[4] T. Killmaier, A. Ramesh Babu, Genetic approach for automatic detection of form deviations of geometrical features for effective measurement strategy, Precision Engineering 27 (4) (2003) 370-381.

[5] Tilo Pfeifera, Syuhei Kurokawab, Stefan Meyera, Derivation of parameters of global form deviations for 3-dimensional surfaces in actual manufacturing processes, Measurement 29 (2001) 179-200.

[6] T.M. Tucker, T.R. Kurefess, Newton methods for parametric surface registration Part I. Theory, Computer-Aided Design 35 (2003) $11_{14}^{107-}$

[7] T.M. Tucker, T.R. Kurefess, Newton methods for parametric surface registration. Part II. Experimental validation, Computer-Aided Design 35 (2003) 115-120.

[8] Woncheol Choi, Thomas R. Kurfess, Jonathan Cagan, Sampling uncertainty in coordinate measurement data analysis, Precision Engineering 22 (1998) 153-163.

[9] M. Mestre, H. Abou-Kandil, Linear prediction of signals uplied to dimensional metrology of industrial surfaces, Measurement 71-72 (1993) 119-134

[10] M. Mestre, Détermination d'intervalles de confiance par prédiction, et optimization de la position des sites de mesure en métrologie dimensionnelle des surface fabriquées, Thèse de doctorat, 1994

[11] W. Choi, T.R. Kurfess, Uncertainty on extreme-fit evaluation for three-dimensional measurement data analysis, Computer-Aided Design 30 (1998) 549-557 
[12] J.M. Linares, J. Bachmann, J.M. Sprauel, Détermination des incertitudes des surfaces associées, Mécanique et industrie 3 (2002) 261-266

[13] J.M. Linares, I. Amara, J.M. Sprauel, Uncertainties of surfaces digitalized by coordinate measuring machines, European Journa of Mechanical and Environmental Engineering 48 (4) (2003) 240246.
[14] M.G. Cox, Measurement uncertainty and the propagation of distributions, in: Proceedings of the 10th International Metrology Congress, Saint Louis, France, 2001 (CD-Rom)

[15] M.G. Cox, A.B. Forbes, J.L. Flowers, P.M. Harris, Least square adjustment in the presence of discrepant data, in: Advanced Mathematical Tools in Metrology VI, Series on Advanced In Mathematics For Applied Science, vol. 66, 2004, pp. 37-51. 